\title{
Can histopathologists diagnose bronchopneumonia?
}

\author{
C R Hunt, E W Benbow, W F Knox, R F T McMahon, L J McWilliam
}

\begin{abstract}
Objectives-To assess histopathologists' ability to accurately diagnose bronchopneumonia, both on naked eye and microscopic examination; to extrapolate from the error rate to determine whether the role of the necropsy in monitoring the epidemiology of clinical error might be compromised.
\end{abstract}

Methods-Review of archival histological sections and necropsy reports from two teaching hospitals in Manchester. The main outcome measures identified were the proportions of macroscopic diagnoses of bronchopneumonia which were confirmed by the original pathologist on histological examination, and which could be confirmed on histological review by independent pathologists, together with the proportion of discrepant diagnoses remedied in the final report by the original pathologist.

Results-Of 279 cases where a macroscopic diagnosis of bronchopneumonia had been noted in the original provisional necropsy report, the original histopathologist described bronchopneumonia in only $206(73.8 \%)$ in the subsequent final report, which took histology into account. Bronchopneumonia could be confirmed on independent histological review in only $193(69 \cdot 2 \%)$ of these cases. The original histopathologist diagnosed 74 cases of bronchopneumonia on histological grounds only, of which only $57(\mathbf{7 7 . 0 \% )}$ could be confirmed on review. Of a total of 160 discrepancies between the original naked eye diagnoses and the final reviewed diagnoses, only $130(81 \cdot 3 \%)$ had been remedied by the original pathologist.

Conclusions-There is a considerable discrepancy rate between naked eye diagnoses of bronchopneumonia at necropsy and diagnoses confirmed on microscopy. If this discrepancy rate is extrapolated to other common lesions, then the role of the necropsy in clinical audit may be compromised. Pathologists need to take steps to monitor and improve their own diagnostic standards.

( $f$ Clin Pathol 1995;48:120-123)

Keywords: Audit, bronchopneumonia, differential diagnosis, necropsy.

The necropsy is often said to be the ultimate audit of clinical diagnosis, ${ }^{12}$ and audit programmes for potentially fatal conditions may be invalid without it. ${ }^{34}$ Many studies show that discrepancies between clinical and necropsy diagnoses are very common, and a recent review quoted discrepancy rates ranging from $11 \cdot 7$ to $33 \cdot 8 \%$ for conditions with a substantial or possible effect on survival. ${ }^{5}$ In some circumstances the discrepancy rates may be even higher. ${ }^{6-10}$

Previously published evaluations of the necropsy have been limited and there has been little emphasis on the accuracy of the interpretations made by the pathologist. It was recently pointed out that the absence of even an estimate of the error rate in necropsy interpretation may limit the value of this procedure in the systematic audit of clinical diagnoses. ${ }^{1112}$ Because of this, we decided to provide such an estimate by reviewing necropsy diagnoses of a very common cause of death, bronchopneumonia.

\section{Methods}

All necropsy reports from Manchester Royal Infirmary for 1989 and 1990 were examined, together with a series of necropsy reports initially selected for another study ${ }^{13}$ at the University Hospital of South Manchester. The latter cases had been randomly selected from necropsies carried out between 1975 and 1984, after stratification for age. Cases were included in the current study if bronchopneumonia, or a synonym of bronchopneumonia, was described in the macroscopic report, the histology report, or both

The histology slides from these cases were retrieved, and all sections of pulmonary tissue were reviewed by two histopathologists, one of consultant status. Any contentious cases were further reviewed by a second consultant histopathologist. To avoid exaggeration of any error rate, a very lenient criterion was used, whereby a scanty infiltrate of neutrophil polymorphs in two or more alveoli was recorded as confirming bronchopneumonia.

Cases were divided into those in which there were discrepancies and those in which there were not. The $\chi^{2}$ test was used to examine the significance of different error rates between consultants and juniors, and between hospital and coroners' necropsies. Cases with discrepancies were further subdivided into those with remedied discrepancies, and those in which discrepancies remained unremedied.

Remedied discrepancies are those cases where the original pathologist corrected either a false positive or false negative naked eye diagnosis when the final report was issued. Unremedied discrepancies are those cases where bronchopneumonia was diagnosed on the original naked eye examination, but where its absence from the subsequent histological
Correspondence to: Dr E W Benbow.

Accepted for publication 18 July 1994 
Table 1 Cases with macroscopy diagnosis of bronchopneumonia in original report: histological confirmation by original histopathologist, and histological confirmation on independent review

\begin{tabular}{llcccl}
\hline Histology & $\begin{array}{l}\text { Presence of } \\
\text { bronchopneumonia }\end{array}$ & UHSM & MRI & Total & Per cent \\
\hline Original & Yes & 91 & 115 & 206 & $73 \cdot 8$ \\
& No & 36 & 37 & 73 & $26 \cdot 2$ \\
Review & Yes & 86 & 107 & 193 & $69 \cdot 2$ \\
& No & 41 & 45 & 86 & $30 \cdot 8$ \\
Total & & 127 & 152 & 279 & \\
\hline
\end{tabular}

UHSM = University Hospital of South Manchester; MRI = Manchester Royal Infirmary.

Table 2 Cases with only microscopy diagnosis of bronchopneumonia by original pathologist

\begin{tabular}{llllll}
\hline Histology & Presence of & & & & \\
bronchopneumonia & UHSM & MRI & Total & Per cent \\
\hline Review & Yes & 40 & 17 & 57 & $77 \cdot 0$ \\
& No & 6 & 11 & 17 & $23 \cdot 0$ \\
\hline
\end{tabular}

UHSM = University Hospital of South Manchester; MRI = Manchester Royal Infirmary.

Table 3 Number of blocks of lung taken for histology from each case

\begin{tabular}{lc}
\hline Number of blocks per case & Number of cases \\
\hline 1 & 46 \\
2 & 147 \\
3 & 82 \\
4 & 50 \\
5 & 11 \\
6 & 8 \\
7 & 6 \\
8 & 3 \\
\hline
\end{tabular}

sections was not taken into account in the final report, together with those examples where a new diagnosis of bronchopneumonia was made on the histological examination, but which could not be confirmed by the reviewers.

\section{Results}

A total of 1091 reports were examined, including 167 hospital necropsies and 578 Coroners' cases from Manchester Royal Infirmary, and 346 hospital necropsies from University Hospital of South Manchester. Bronchopneumonia was mentioned in the macroscopic findings in 279 of these, but confirmed in the original histology report in only 206; only 193 diagnoses of bronchopneumonia could be confirmed on histological review by independent pathologists (table 1). In a further 74 cases bronchopneumonia was mentioned for the first time in the original histology report; of these cases, only 57 could be confirmed on histological review (table 2). In these 353 cases the mean number of blocks taken was $2 \cdot 7$, with a range of 1 to 8 ; in $46(13.0 \%)$ cases, only one block was taken (table 3 ). There was a slight tendency, not reaching statistical significance, for discrepancies to be greater in necropsies carried out for the Coroner than in those carried out at the request of the hospital clinicians $\left(\chi^{2}=0 \cdot 583, \mathrm{DF}=1, \mathrm{p}=0 \cdot 445\right)$. Subconsultant staff have a statistically significant tendency to

Table 4 Comparison between consultants and junior staff

\begin{tabular}{lllll}
\hline $\begin{array}{l}\text { Grade of } \\
\text { pathologist }\end{array}$ & $\begin{array}{l}\text { Confirmed } \\
\text { diagnosis }\end{array}$ & Per cent & $\begin{array}{l}\text { Discrepant } \\
\text { diagnosis }\end{array}$ & Per cent \\
\hline Consultant & 103 & $79 \cdot 8$ & 26 & $20 \cdot 2$ \\
Junior & 147 & $65 \cdot 6$ & 77 & $34 \cdot 4$ \\
\hline
\end{tabular}

$\chi^{2}=7 \cdot 337, \mathrm{DF}=1, \mathrm{p}=0.007$
Table 5 Examples of correct histological diagnoses when original diagnoses could not be confirmed

Diagnosis

Chronic airways obstruction

Normal lung

Intra-alveolar haemorrhage

Aspiration (without inflammation)

Bone marrow embolism

Secondary invasive aspergillosis

Leukaemic infiltrate

Pleural inflammation

Pulmonary oedema

Infarction

make more discrepant diagnoses than consultant staff (table 4). Some of the correct histological diagnoses, in those cases in which bronchopneumonia could not be confirmed on review, are shown in table 5 .

In 130 cases discrepancies had been corrected by the original pathologist, but in 30 cases they had not. Neither category of discrepancy was particularly associated with grade of pathologist, or with type of necropsy. Unremedied discrepancies, as opposed to remedied discrepancies, were not particularly associated with grade of pathologist $\left(\chi^{2}=\right.$ $0.049, \mathrm{DF}=1, \mathrm{p}=0.825)$, and although they were more often associated with Coroners' than with hospital necropsies, this correlation also failed to reach statistical significance $\left(\chi^{2}=\right.$ $1 \cdot 848, \mathrm{DF}=1, \mathrm{p}=0 \cdot 174)$.

\section{Discussion}

The literature contains many papers, reviews, and editorials which extol the advantages of the necropsy, but many are descriptive evaluations without any quantitative information. In others the authors have used the necropsy as the gold standard of clinical diagnoses, and there are many publications which describe discrepancy rates between clinical and necropsy diagnoses ${ }^{5}$; generally, these include an implicit assumption that the necropsy diagnosis must be correct. In a recent and searching review, however, Saracci ${ }^{12}$ pointed out that the literature contains no satisfactory information about the accuracy of necropsy diagnoses and suggested that this deficiency compromises the role of the necropsy in systematic clinical audit. Pathologists have also bemoaned the absence of published quality assurance data on the necropsy. ${ }^{14}$

Saracci goes on to provide a detailed description of how the deficiency, in his view, should be rectified, and outlines three major requirements: comprehensive peer review, long term standardisation of procedures, and appropriate sampling. ${ }^{12}$ These requirements would, under current working conditions, be difficult or impossible to fulfil for most departments. Peer review, if carried out conscientiously, would take up too much time, and the requirement for standardised procedures would substantially increase workloads, especially if a rigid system of histological sampling were adopted. Proper sampling of cases, whereby a random selection of all patients dying in both hospitals and the community would be subjected to necropsy is not feasible under current legal provisions, and would be 
unacceptable to the general public. Because of these difficulties, we looked for a paradigm whereby retrospective review of archival necropsy slides might be used as a rough guide to the order of magnitude of the error rate at necropsy. The diagnosis of certain conditions could not, because of their nature, be audited by histological review-for instance, bony fractures and massive pulmonary embolism do not require histological confirmation. Other conditions, such as myocardial infarction, were excluded because of the difficulty of establishing minimum histological criteria for early lesions, and many conditions are not seen sufficiently often for a large number of cases to be accumulated for study. We therefore chose to audit diagnoses of bronchopneumonia, a common disease which is usefully characterised by histology even when macroscopic diagnosis seems irrefutable, and for which a minimum histological criterion can be easily established.

One possible criticism of the design of our study is that discrepancies may represent the effects of inappropriate or casual tissue sampling by the original pathologist, rather than true errors of interpretation. This criticism is highlighted by our observation that only one block of lung was taken in $13 \%$ of cases, although most pathologists would regard two blocks as a minimum even in cases where there is no reason to suspect pulmonary disease. Strictly speaking, therefore, we cannot categorically state that all the discrepancies revealed by our study truly reflect errors of diagnosis. Incorrect sampling, however, prevents eventual correction at histology and is a crucial error. Our study might also be criticised because we chose not to review those cases where there was no mention of bronchopneumonia in any component of the original report: to have done so would have been prohibitively time-consuming. Because of this, we cannot calculate true sensitivity and specificity. Despite these criticisms, and despite the fact that our study cannot approach Saracci's high standards, we feel that our results are important. The discrepancies that we found fall readily into two distinct categories: those remedied by the original pathologist and those which remained unremedied even after microscopy. These two categories raise very different issues.

Remedied discrepancies were present in 130 cases, representing $36 \cdot 8 \%$ of the 353 cases studied and $11.9 \%$ of the original population of 1091 reports from which the study cases were derived. Bearing in mind that we studied only one kind of lesion, it is likely that there were many other discrepancies capable of correction by histological examination. This supports a recent recommendation, made by a Working Party representing a triumvirate of Royal Colleges, ${ }^{15}$ that histology should be carried out at all necropsies. Although routine necropsy histology may be thought by some to be too expensive ${ }^{16}$ or too time-consuming, ${ }^{1718}$ it seems irresponsible to turn down the opportunity to correct such a large number of erroneous interpretations. If used in a constructively self-critical way, histology provides a form of continuing personal audit which one would expect to improve and maintain standards. Coroners' cases are a particular problem because remuneration is generally not forthcoming to fund histology, unless it is necessary to establish the cause of death, and only if the Coroner sanctioned its use at the time of the original autopsy. ${ }^{19}$ Although some recent reports indicate that histological examination is so much an intrinsic part of the necropsy that it is permissable to retain tissue for histology even if histological examination is not vital for establishing the cause of death, ${ }^{2021}$ recent controversy about this matter ${ }^{2022}$ may inhibit pathologists from carrying out full histological examinations in more than a small minority of Coroners' necropsies.

The second issue is of more fundamental importance when we consider the role of the necropsy in clinical audit, with 30 cases showing unremedied discrepancies. This represents $8.5 \%$ of our study cases and $2 \cdot 7 \%$ of our original population. These proportions are probably far greater than most pathologists would have anticipated, and are greater than the maximum error which Saracci thought would be acceptable in formal epidemiological studies of discrepancies between clinical and pathological diagnoses.

The reasons for the errors in our series, particularly in the interpretation of histology, are difficult to determine: in numerous cases bronchopneumonia had been diagnosed where no inflammatory infiltrate of any kind could be found. In other cases possible confounding lesions included a leukaemic infiltrate, bone marrow embolism, and a terminal aspiration without inflammatory infiltrate. The errors of macroscopic diagnosis are, for the most part, more easily understood: lesions such as those of early adult respiratory distress syndrome, which was common in our series, readily simulate the macroscopic changes of pneumonia. In other cases, however, there was no pulmonary lesion on the histology slides, or the pulmonary lesion had little resemblance to bronchopneumonia, such as emphysema, pulmonary embolism, or pulmonary infarction.

Our results suggest that pathologists may use bronchopneumonia as a diagnosis of convenience, in much the same fashion as clinicians filling out death certificates, and it may be that some are reluctant to change a macroscopic diagnosis, implicitly confessing a previous error. We have shown that histology is capable of correcting a large proportion of necropsy diagnoses, and we support the recommendation that it should be carried out in all cases. The error rate on histological examination itself is unacceptably high, though the observation that consultants perform better than juniors indicates that at least some of the errors are avoidable, and suggests that more emphasis should be placed on histological interpretation when pathologists are trained in necropsy practice. Further studies of discrepancy rates between clinical and necropsy diagnoses should distinguish between those made with the benefits of histology, and those made without. 
1 Gau G. The ultimate audit. BMF 1977;1:1580-2.

2 Gambino SR. The autopsy: the ultimate audit. Arch Pathol Lab Med 1984;108:444-5.

3 Hill RB, Anderson RE. Is a valid quality assessment program possible without the autopsy? Hum Pathol 1988;19: 1125-6.

4 Underwood JE, Cotton DWK, Stephenson TJ. Audit and necropsy. Lancet 1989;i:442.

5 Hill RB, Anderson RE. Missed diagnosis: toward an understanding of diagnostic discrepancies. The autopsymedical practice and public policy. Stoneham, Massachusetts: Butterworth, 1988: 67-90.

6 Berg JW, Downing A, Lukes RJ. The prevalence of undiagnosed cancer of the large bowel found at autopsy in different races. Cancer 1970;25:1076-80.

7 Cameron HM, McGoogan E. A prospective study of 1152 hospital autopsies: II. Analysis of inaccuracies in clinical diagnoses and their significance. $f$ Pathol 1981;133:285300 .

8 Zarling EJ, Sexton H, Milnor P. Failure to diagnose acute myocardial infarction. The clinicopathological experience at a large community hospital. $\mathfrak{J A M A} 1983$; perience at a
$\mathbf{2 5 0}: 1177-81$.

9 Battle RM, Pathak D, Humble G, Key CR, Vanatta PR, Hill $\mathrm{RB}$. Factors influencing discrepancies between premortem and postmortem diagnoses. $\mathscr{f} A M A$ 1987;258:339-44.

10 Tench DW, Benbow SM, Benbow EW. Do old age psychiatrists miss physical illnesses? Int $\mathcal{f}$ Geriatr Psychiat 1992;7:713-18.

11 Saracci R. Autopsy as a yardstick of diagnosis: an epidemiologist's remarks. In: Riboli E, Delendi M, eds. Aut- opsy in epidemiology and medical research. Lyon: IARC, 1991:185-96.

12 Saracci $R$. Is necropsy a valid monitor of clinical diagnosis performance? BMF 1991;303:898-900.

13 Catania J, McWilliam L, O'Neill PA, Roberts M. Is accuracy of death certification influenced by age of deceased? Age Ageing 1991;20(Suppl 1):28.

Ageing 1991;20(Suppl 1):28.
14 Anderson RE, Hill RB, Gorstein F. A model for the autopsybased quality assessment of medical diagnostics. Hum Pathol 1990;21:174-81.

15 Joint Working Party of the Royal College of Pathologists, the Royal College of Physicians of London and the Royal College of Surgeons of England. The autopsy and audit. London: Royal College of Pathologists, 1991.

16 Reid WA. Cost effectiveness of routine postmortem histology. F Clin Pathol 1987;40:459-61.

17 Roberts WC. The autopsy: its decline and a suggestion for its revival. $N$ Engl f Med 1978;299:332-8.

18 Saladino AJ, Dailey ML. The problem-oriented postmortem examination. Am $\mathcal{f}$ Clin Pathol 1978;69:253-7.

19 Timperley WR Retention of post mortem material. London: Bulletin of the Royal College of Pathologists, 1990;(72)19. 20 Popper SL. Retention of post mortem material. London: (73) $21-2$.

21 Skegg PDG. The use of corpses for medical education and research: the legal requirements. Med Sci Law 1991;31 345-54.

22 Timperley WR. Retention of post mortem material-reply. London: Bulletin of the Royal College of Pathologists, $1991 ;(73) 22$. 